\title{
Temperature Dependence of Retention Factor of Saccharides on Hydrophobic Resin
}

\author{
Utano AIMOTO, Takashi KOBAYASHI, and Shuji ADACHI ${ }^{\dagger}$ \\ Division of Food Science and Biotechnology, Graduate School of Agriculture, \\ Kyoto University, Sakyo-ku, Kyoto 606-8502, Japan
}

\begin{abstract}
Retention factors of galactose, glucose, trehalose, and maltotriose for a column packed with hydrophobic resins were measured using water as the eluent in the temperature range of 0.5 to $150^{\circ} \mathrm{C}$. The van't Hoff plots of the retention factors were nonlinear, indicating that hydrophobic interactions between the resin and solutes occurred. The temperature dependence of the retention factor could be analyzed based on Kirfhoff's law and the enthalpy change for distribution was calculated.
\end{abstract}

Key words: retention factor, hydrophobic interaction, saccharide

\section{Introduction}

A high performance liquid chromatography, HPLC, analysis is carried out at an appropriate temperature. The heat labile substances are separated at relatively low temperature. On the other hand, separation of the stable substances is usually performed at room temperature or higher temperature. Separation at high temperature is called high temperature liquid chromatography and is abbreviated as HTLC. Because some physicochemical parameters playing an important role in HPLC, such as viscosity, mobile phase polarity and diffusivity, depend on the temperature, it is known that the temperature is an important factor not only for the stability of the solutes but for their separability.

The temperature effect on the distribution behavior in HPLC should be examined for better understanding of the separation phenomena and kinetics. Some reports discuss the temperature dependence of the theoretical plate number [1-4] and the distribution coefficient [5] in HTLC. Furthermore, the temperature dependence of the kinetic parameters of biologically active peptides in the temperature range of -15 to $5^{\circ} \mathrm{C}$ [6], of sugars in the temperature range of 5 to $60^{\circ} \mathrm{C}$ [7], and of amino acids in the temperature range of 20 to $55^{\circ} \mathrm{C}$ [8] has been reported. However, knowledge of the distribution coefficients or the retention factors at much higher temperatures is still insufficient.

(Received 11 Jul. 2011: accepted 27 Oct. 2011)

† Fax: 075-753-6285, E-mail: adachi@kais.kyoto-u.ac.jp
Water which is extensively used as the eluent of the liquid chromatography is known to show unique features at high temperature under sufficient pressure to maintain its liquid state; e.g., the temperature dependence of the solubility of saturated fatty acids in water at temperatures higher than $160^{\circ} \mathrm{C}$ obeyed the van't Hoff equation but at lower temperatures, the dependence deviated from the equation [9], because the formation of the hydrogen bonds in water largely depends on temperature. This effect would play an important role when liquid chromatography, in which the separation occurs mainly by hydrophobic interaction, is conducted using water as the eluent at high temperature.

The aim of this study is to measure the retention factors of monosaccharides (galactose and glucose), disaccharide (trehalose), and trisaccharide (maltotriose), which would have moderate hydrophobicity, on the hydrophobic resin using water as the eluent in the temperature range of 0.5 to $150^{\circ} \mathrm{C}$.

\section{Materials and Methods}

\subsection{Materials}

The high temperature endurance column, in which the hydrophobic resin (octadecyl coated; particle diameter, $4 \mu \mathrm{m}$; pore size, about $25 \mathrm{~nm}$ ) was packed, (ET-RP1 4D, 150 mm L. ×4.6 mm I.D., Shodex, Tokyo, Japan) was used in this study. Deuterium oxide (purity, 99.96\%), $\mathrm{D}^{-(+)}$-galactose, $\mathrm{D}^{-(+)}$-glucose, and maltotriose of analytical grade were purchased from Wako Pure Chemical 
Industries (Osaka, Japan), and trehalose was from Hayashibara Biochemical Laboratories (Okayama, Japan). Trehalose was used as the solute because it was more stable than maltose at high temperature [10]. Pure water was used as an eluent.

\subsection{Estimation of retention factors}

A column was installed in a handmade thermal controller. Column temperature was adjusted to the objective temperature $\pm 0.3^{\circ} \mathrm{C}$ during the experiments. An eluent was fed to the column with an LC-10AT $\mathrm{VP}$ pump (Shimadzu, Kyoto, Japan). To prevent vaporization of the eluent, the pressure of the system was maintained at 3.0 $\mathrm{MPa}$ using a backpressure regulator (Upchurch Scientific, Oak Harbor, WA, USA). The column effluent was introduced into a cooling tube and allowed to flow into a refractive index detector (RID-6A, Shimadzu) to determine the solute concentration. The chromatogram was recorded using Chromatopac C-R8A (Shimadzu). The dead volume was determined by removing the column and measuring the elution time of galactose.

Galactose, glucose, trehalose, and maltotriose were separately dissolved in water at $0.5 \%$ (w/v). A $10-\mu \mathrm{L}$ sample was applied to the column through a sample injector. Column temperature was regulated at a temperature in the range of 0.5 to $150^{\circ} \mathrm{C}$, and the flow rate of the eluent was changed in the range of 0.50 to 0.80 $\mathrm{mL} / \mathrm{min}$.

The total void volume, $V_{0}$, which means sum of bed voidage and resin pore volume, was determined by the elution time of deuterium oxide. The bed voidage, $\varepsilon_{\mathrm{b}}$, is usually estimated from the elusion behavior of a macromolecule, such as dextran, which is too large to penetrate into the pores [11]. However, the pore size of the resin used in this study is very large (about $25 \mathrm{~nm}$ ), and it was impracticable to find an adequate macromolecule for estimating the bed voidage. Therefore, the bed voidage value was assumed to be 0.30 .

\section{Results and Discussion}

Figure 1 shows the elution curves of the four solutes obtained at $20^{\circ} \mathrm{C}$ and $90^{\circ} \mathrm{C}$ using water as the eluent at $0.45 \mathrm{~mL} / \mathrm{min}$. At $20^{\circ} \mathrm{C}$, the low molecular mass solute eluted faster than the high molecular mass solute, whereas all the solutes eluted at almost the same elution time at $90^{\circ} \mathrm{C}$. A similar tendency of faster elution at higher temperature was observed for all the solutes over the whole tested temperature range.
The distribution equilibrium of the solute between the mobile phase and the stationary one is related to the free energy change, $\Delta G$, associated with solute distribution from the mobile phase to the stationary one by Eq. (1)

$$
\ln K=-\frac{\Delta G}{R T}=-\frac{\Delta H}{R T}+\frac{\Delta S}{R}
$$

where $K$ is the distribution coefficient, $T$ is the absolute temperature, $R$ is the gas constant, and $\Delta H$ and $\Delta S$ are the enthalpy and entropy changes, respectively, for solute distribution from the mobile phase to the stationary phase. The retention factor, $k$, which shows the magnitude of retention of the solute, is given by Eq. (2)

$$
k^{\prime}=\frac{V_{\mathrm{R}}-V_{0}}{V_{0}}
$$

where $V_{R}$ is the retention volume of the solute. Since the retention factor is related to $K$ and the volume phase

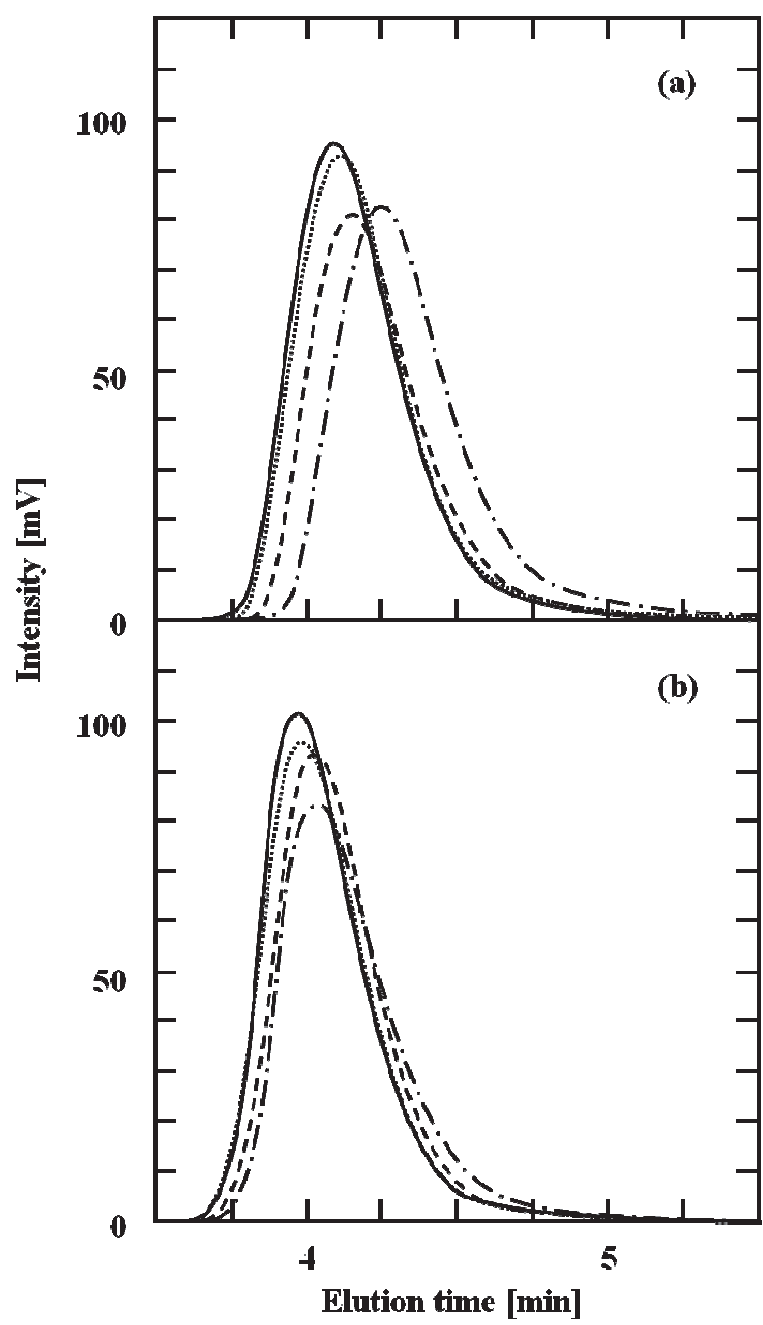

Fig. 1 Elution curves of (-) galactose, $(\cdots \cdots)$ glucose, (---) trehalose, $(-\cdot-)$ maltotriose at (a) $20^{\circ} \mathrm{C}$ and (b) $90^{\circ} \mathrm{C}$ using water as an eluent at $0.45 \mathrm{~mL} / \mathrm{min}$. 
ratio, $\varphi$, which is the volume ratio of the stationary to the mobile phase:

$$
k^{\prime}=K \varphi
$$

Therefore, Eq. (1) can be transformed into Eq. (4).

$$
\ln k^{\prime}=-\frac{\Delta H}{R T}+\frac{\Delta S}{R}+\ln \varphi
$$

The volume of the stationary phase cannot be determined, so the value of $\varphi$ assumed to be equal to $\left(1-\varepsilon_{\mathrm{b}}\right) /$ $\varepsilon_{\mathrm{b}}$. This assumption, of course, contains some errors in estimation of entropy change because the value of $\varphi$ is larger than the values of $k$ of each solutes. However, the tendency of enthalpy change at different temperatures could be estimated.

When enthalpy and entropy changes are temperatureinvariant, the plots of $\ln k^{\prime}$ or $\ln K$ versus $1 / T$ (van't Hoff plot) are linear and allow their direct evaluation $[5,8]$. Figure 2 shows the temperature dependence of the $k^{\prime}$ values of the solutes in the temperature range of 0.5 to $150^{\circ} \mathrm{C}$. The plots for each solute, however, lay on a curve in the tested temperature range. When $\Delta H$ and $\Delta S$ are temperature-dependent, the integrated form of Kirchoffs law is used to evaluate the thermodynamic quantities $[12,13]$. If the heat capacity change, $\Delta C_{\mathrm{p}}$, is invariant with temperature, the dependences of $\Delta H$ and $\Delta S$ on the temperature are given by Eqs. (5) and (6), respectively.

$$
\Delta H=\Delta C_{\mathrm{p}}\left(T-T_{\mathrm{H}}\right)
$$

\section{Column temperature $\left[{ }^{\circ} \mathrm{C}\right.$ ]}

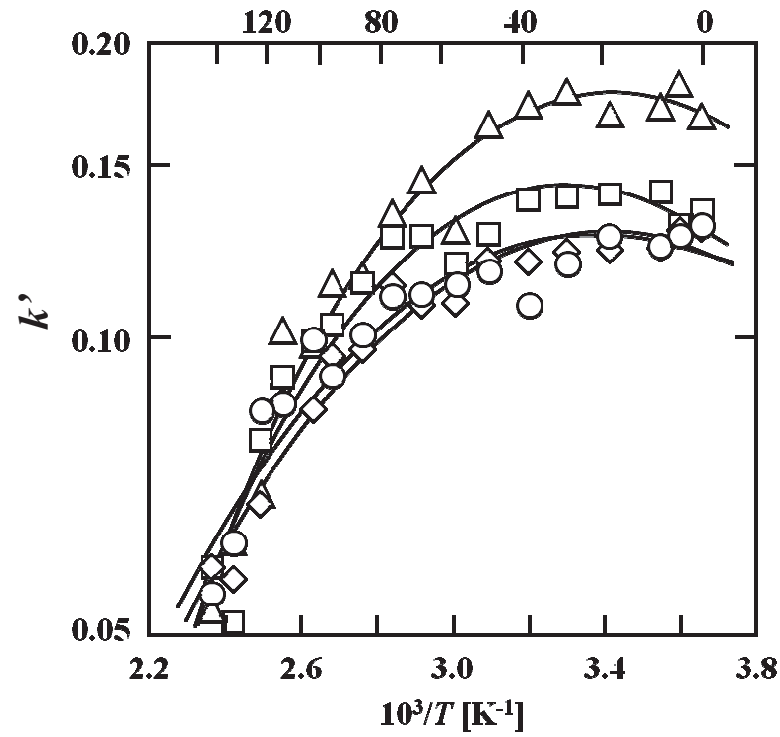

Fig. 2 Temperature dependence of the retention factors of $(\diamond)$ galactose, $(\bigcirc)$ glucose, $(\square)$ trehalose, and $(\triangle)$ maltotriose. Solid curves represent the best fits of Eq. (7) to the data.

$$
\Delta S=\Delta C_{\mathrm{p}} \ln \left(T / T_{\mathrm{S}}\right)
$$

where $T_{\mathrm{H}}$ and $T_{\mathrm{S}}$ are reference temperatures at which $\Delta H$ and $\Delta S$ are zero. Substitution of Eqs. (5) and (6) into Eq. (4) gives the following equation:

$$
\ln k^{\prime}=\frac{\Delta C_{\mathrm{p}}}{R}\left(\frac{T_{\mathrm{H}}}{T}-\ln \frac{T_{\mathrm{S}}}{T}-1\right)+\ln \varphi
$$

Equation (7) allows the evaluation of the three parameters, $T_{\mathrm{H}}, T_{\mathrm{S}}$ and $\Delta C_{\mathrm{p}}$, from nonlinear van't Hoff plots by a least-squares fitting using the Solver in Microsoft Excel $2007^{\circledR}$.

The temperature dependence of the retention factor could be fitted to Eq. (7) with $\mathrm{R}^{2}>0.91$ for each solute (solid curves in Fig. 2) and the estimated values of $T_{\mathrm{H}}, T_{\mathrm{S}}$ and $\Delta C_{\mathrm{p}}$ are shown in Table 1 . The $\Delta H$ values were calculated at different temperatures for the solutes (Fig. 3) using Eq. (5), and the $\Delta H$ decreased with increasing temperature. This result showed that the distribution of saccharides from the eluent to the hydrophobic resin was enthalpy-driven in the high temperature.

Table 1 Estimated values of $T_{\mathrm{H}}, T_{\mathrm{S}}$ and $\Delta C_{\mathrm{p}}$ in Eq. (7)

\begin{tabular}{ccccc}
\hline & Galactose & Glucose & Trehalose & Maltotriose \\
\hline$T_{\mathrm{H}}[\mathrm{K}]$ & 294 & 295 & 304 & 294 \\
$T_{\mathrm{S}}[\mathrm{K}]$ & 237 & 233 & 262 & 258 \\
$\Delta C_{\mathrm{p}}[\mathrm{J} /(\mathrm{K} \cdot \mathrm{mol})]$ & -112 & -102 & -157 & -163 \\
\hline
\end{tabular}

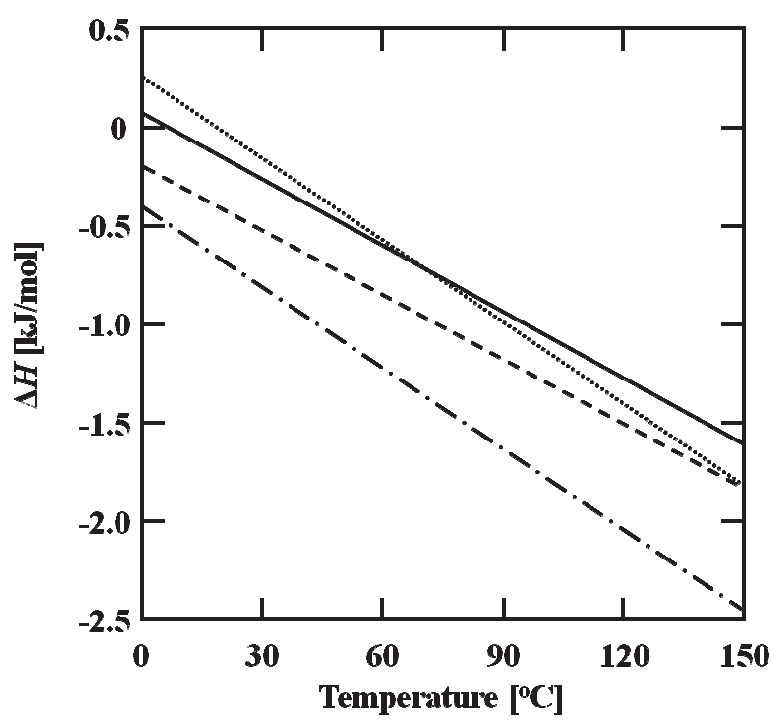

Fig. 3 Temperature dependence of $\Delta H$ for distribution of $(-)$ galactose, $(\cdots \cdots)$ glucose, (---) trehalose, and $(-\cdot-)$ maltotriose from the mobile phase to the stationary phase. 
At low temperature, the retention factor was larger for the solute of larger molecular mass. This order of increasing retention factor was in the same order of increasing hydrophobicity: monosaccharide $<$ disaccharide $<$ trisaccharide [14]. This would be ascribed to the formation of hydrophobic interactions between the resin and the saccharides. Considering the structures of saccharides, Janado et al. reported that the hydrophobicity of galactose was weaker than glucose [14]. However, no apparent differences were measured in this study. Exclusion of the hydrophobic moieties of saccharides from water which is associated by hydrogen bonds induces the hydrophobic interactions. Almost the same retention factors of the saccharides at high temperatures would indicate the reduction of hydrogen bonds in water at the temperatures.

\section{References}

1) Y. Yang; A model for temperature effect on column efficiency in high-temperature liquid chromatography. Anal. Chim. Acta., 558, 7-10 (2006)

2) J. Li, Y. Hu, P. W. Carr; Fast separations at elevated temperatures on polybutadiene-coated zirconia reversed-phase material. Anal. Chem., 69, 3884-3888 (1997)

3) D. Guillarme, S. Heinisch, J. L. Rocca; Effect of temperature in reversed phase liquid chromatography. J. Chromatogr. A, 1052, 39-51 (2004)

4) J. Li, P. W. Carr; Effect of temperature on the thermodynamic properties, kinetic performance, and stability of polybutadiene-coated zirconia. Anal. Chem., 69, 837-843 (1997)

5) S. Kikuchi, T. Kobayashi, S. Adachi; Temperature dependences of the distribution coefficients of hydrophobic solutes onto porous styrene divinylbenzene resin for the eluent with a low methanol content. J. Biosci. Bioeng., 106, 208-210 (2008)
6) D. E. Henderson, J. A. Mello; Physicochemical studies of biologically active peptides by low-temperature reversed-phase high-performance liquid chromatography. J. Chromatogr. A, 499, 79-88 (1990)

7) C. B. Ching, K. Hidajat, M. N. Rathor; Chromatographic evaluation of sorption and diffusion characteristics of glucose, maltose and maltotriose in silica gels. J. Chromatogr. A, 463, 261-270 (1989)

8) M. S. Uddin, K. Hidajat, C. B. Ching; Liquid chromatographic evaluation of equilibrium and kinetic parameters of large molecule amino acids on silica gel. Ind. Eng. Chem. Res., 29, 647-651 (1990)

9) P. Khuwijitjaru, S. Adachi, R. Matsuno; Solubility of saturated fatty acids in water at elevated temperatures. Biosci. Biotechnol. Biochem., 66, 1723-1726 (2002)

10) T. Oomori, S. H. Khajavi, Y. Kimura, S. Adachi, R. Matsuno; Hydrolysis of disaccharides containing glucose residue in subcritical water. Biochem. Eng. J., 18, 143-147 (2004)

11) K. Hashimoto; "Chromatographic Separation Engineering (Kuromato Bunnri Kougaku)”, Baifukan, Tokyo, Japan, 2005, p. 67.

12) D. Haidacher, A. Vailaya, C. Horváth; Temperature effects in hydrophobic interaction chromatography. Proc. Natl. Acad. Sci., 93, 2290-2295 (1996)

13) R. L. Baldwin; Temperature dependence of the hydrophobic interaction in protein folding. Proc. Natl. Acad. Sci., 83, 8069-8072 (1986)

14) M. Janado, Y. Yano; Hydrophobic nature of sugars as evidenced by their differential affinity for polystyrene gel in aqueous media. J. Solution Chem., 14, 891-902 (1985) 